\title{
DIRECT PRODUCT OF DIVISION RINGS AND A PAPER OF ABIAN
}

\author{
M. CHACRON ${ }^{1}$
}

Abstract. It is shown that the rings under the title admit an order-theoretical characterization as in the commutative case studied by Abian.

Introduction. Let $R$ be an associative ring equipped with the binary relation ( $\leqq$ ) defined by $x \leqq y$ if and only if $x y=x^{2}$ in $R$. In this paper, it is shown that (ฏ) is an order relation on $R$ if and only if, $R$ has no nilpotent elements $(\neq 0)$. Conditions on the binary relation $(\leqq)$ in order that $R$ split into a direct product of division rings are then studied in the light of Abian's result [1, Theorem 1]. Using similar argumentation and using certain subdirect representation of rings with no nilpotent elements, one obtains a complete similarity with the commutative case (yet, no extra complication in the computations).

Conventions. $R$ is an associative ring which is, unless otherwise stated, with no nilpotent elements (other than 0 ). As a result of [2], $R$ can be embedded into a direct product of skewdomains, $R \rightarrow \prod_{\iota} \in I R_{\text {، }}$ (that is to say, rings $R_{\iota}$ having no one-sided divisors of zero). The former embedding is fixed throughout the paper. It is therefore legitimate to identify any element $x$ in $R$ with the family consisting of all its projections $\left(x_{\mathfrak{\imath}}\right)_{\mathfrak{t}} \in \boldsymbol{I}$. Finally, all definitions in [1] are extended (verbatim) to the present case (of a noncommutative ring $R$ ) and are freely used throughout.

In this paper we offer the following generalization to the noncommutative case of Abian's result [1, Theorem 1].

THEOREM. Any ring $R$ equipped with its binary relation (ฏ) defined by $a \leqq b$, if and only if $a b=a^{2}$, is isomorphic to a direct product of division rings if and only if (ฏ) is an order relation on $R$ such that $R$ is hyperatomic and orthogonally complete (in the sense of Abian).

The 'only if' is just a combination of the forthcoming Lemma 2

Received by the editors June 17, 1970.

AMS 1969 subjec tclassifications. Primary 1646; Secondary 0685.

Key words and phrases. Noncommutative rings, rings having no nilpotent elements $\neq 0$, subdirect product, subdirect representation, hyperatomic, idempotent hyperatom, supremum.

${ }^{1}$ This research has been supported partly by a summer fellowship (SRI, Branch at Université Laval) partly by grant A4807 of the NRC of Canada.

Copyright @ 1971, American Mathematical Society 
and a partial duplication of Abian's proof. (See first part of the proof of $[1$, Theorem 1].) The 'if' breaks into several lemmas some which are of independent interest.

LEмма 1. If $a \leqq b$ is an order relation on a ring $R$, then $R$ has no nilpotent elements $(\neq 0)$.

Proof. Assume $x^{2}=0$ in $R$. Then $x \leqq 0$. However 0 is the least element under $\leqq$. Therefore $x=0$.

Lемма 2. If $R$ has no nilpotent elements, then (ฏ) is a (multiplicatively) permissible (that is to say, $a \leqq b$ implies $a c \leqq b c$ and $c a \leqq c b$ for all $c \in R$ ) order relation on $R$.

Proof. Using the mentioned embedding of $R$ (see Conventions), it suffices to show the result for a skewdomain. In this case the occurrence $a b=a^{2}$, is equivalent with $a=0$ or $a=b$. Now the latter occurrence defines a binary relation which is obviously a permissible order relation on $R$. Lemma 2 is established.

Some known properties of rings without nilpotents $R$ which follow at once from the considered embedding of $R$, are collected without proof in the next lemma.

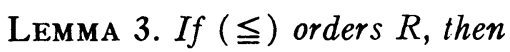

(1) Any idempotent element $e\left(=e^{2}\right)$ of $R$ is in the center of $R$, and ex $=x e \leqq x$ for all $x \in R$.

(2) For any $a, x \in R$ (i) $x^{2} a=x^{2}$ implies $x a=x$; (ii) $x^{2} a=x$ implies $x a x=x$; (iii) $x^{2} a=0$ implies $x a=0$.

We assume henceforth that $R$ is ordered, or equivalently (Lemmas 1 and 2 ), that $R$ has no nilpotent elements. Along the lines of the proof of [1, Theorem 1] let us now show

LemmA 4. Let $a$ be an hyperatom of $R$. For any $r$ such that ar $\neq 0$, ar is an hyperatom.

Proof. Let $x \leqq a r$ in $R$. By definition [1, Definition 1, (6)], there is $s$ so that $x s \leqq a r s=a$. As $x \leqq a r, x^{2}=x a r$ implies $x^{2} s=x a r s=x a$, and $x^{2}=(x a) r=\left(x^{2} s\right) r=x^{2}(s r)$. Then (Lemma 3, (2)(i)) $x=x(s r)$ $=(x s) r$. As $a$ is an hyperatom, either $x s=0$ and so, $x=(x s) r=0$, or $x s=a$ in which case $x=a r$. Finally, assume $(a r) y \neq 0$ for some given $y$. Then, for $t=t^{\prime} r$ with $t^{\prime}$ so that $a(r y) t^{\prime}=a$, we get $(a r) y t=a(r y) t^{\prime} r=a r$ proving thereby that $a r$ is an hyperatom.

Leмма 5. Let $x \neq 0$ in $R$. Assume that $q \leqq x$ for some hyperatom $q \neq 0$. Then there is an idempotent hyperatom $e$ such that ex $\neq 0$. 
Proof. As $q \neq 0, q^{2} \neq 0$. Then $q^{2} s=q$ for some $s$. It follows (Lemma 3 , (2) (iii)) that $q s q=q$. Set $e=q s=s q$. As $q \neq 0, e \neq 0$ and by Lemma $4, e$ is an idempotent hyperatom. Evaluating $e x$, we get $e x=q s x=s q x$ $=s q^{2}=q s q=q \neq 0$.

LEMмA 6. The set $E=\left\{e_{\iota}, \iota \in I\right\}$ of all idempotent hyperatoms of $R$ is an orthogonal set and each of its elements $e_{\downarrow}$ generates a division ring $D_{\iota}=e_{\iota} R$.

Proof (SKeTChed). By definition of an hyperatom $e$ such that $e=e^{2}$, and by property (1) in Lemma 3 .

LEMMA 7. If $R$ is hyperatomic, then $f=x \rightarrow\left(e_{\imath} x\right)_{\imath} \in I$ is a monomorphism from $R$ into a direct product of division rings $D_{\mathfrak{\imath}}=e_{\mathfrak{\imath}} R$.

Proof. It is an immediate consequence of Lemmas 5 and 6.

Lемма 8. The embedding in Lemma 7 has the following properties:

(1) Each factor $D_{\imath}=e_{\imath} R$ is a skewdomain.

(2) If $\left(a^{(\alpha)}\right)_{\alpha \in A}$ is any family of elements of $R$ having a supremum a in $R$ then for any fixed $\iota \in I, a_{\iota}^{(\alpha)}=0$ for all $\alpha \in A$ implies $a_{\iota}=0$.

Proof. Let $\left(a^{(\alpha)}\right)_{\alpha \in A}$ be a family of elements in $R$ admitting supremum $a$ in $R$. Let $\iota \in I$ such that $a_{\imath}^{(\alpha)}=0$ for all $\alpha \in A$. To prove that $a_{\imath}=0$. Here $a_{\imath}=e_{\imath} a$. Set $a^{\prime}=a-e_{\imath} a$. As $E$ is orthogonal,

$$
\begin{aligned}
& a_{\mu}^{\prime}=a_{\mu} \text { if } \mu \neq \iota, \\
& =0 \text { if } \mu=\imath \text {. }
\end{aligned}
$$

Then $a_{\mu}^{(\alpha)} \leqq a_{\mu}^{\prime}$ for all $\mu \in I$. Consequently, $a^{(\alpha)} \leqq a^{\prime}$ for all $\alpha \in A$. Then $a \leqq a^{\prime}$, that is to say, $a\left(a-e_{\triangleleft} a\right)=a^{2}$, if and only if, $a^{2} e_{\iota}=0$, equivalently $a e_{\imath}=0$, equivalently, $a_{\imath}=0$.

LEмма 9. If $R$ admits at least one imbedding as in Lemma 8, then $R$ has the following property:

(A) For any family $\left(a^{(\alpha)}\right)_{\alpha \in A}$ of elements of $R$ admitting a supremum in $R$, and any $b \in R,\left(b a^{(\alpha)}\right)_{\alpha \in A}$ admits a supremum in $R$ equal to $b\left(\sup _{\alpha} a^{(\alpha)}\right)$.

Proof. As ( $\leqq$ ) is left permissible, $a^{(\alpha)} \leqq a$ implies $b a^{(\alpha)} \leqq b a$ for any $\alpha \in A$, and $b a$ is an upper bound of $\left\{b a^{(\alpha)}, \alpha \in A\right\}$. Also, $b a$ is the least upper bound. For let $b a^{(\alpha)} \leqq u$ in $R$. If $b a \leqq u$ were not true, then for some $\iota \in I, b_{\imath} a_{\imath} \neq u_{\imath}$. Then $a_{\imath} \neq 0$, and $a_{\imath}^{\left(\alpha_{0}\right)}$ for some $a_{0} \in A$ follows. As $a_{\imath}^{\left(\alpha_{0}\right)} \leqq a_{\imath}$, in the skewdomain $D$ เ we must have $a_{\imath}^{\left(\alpha_{0}\right)}=a_{\imath}$ (see Lemma 2 and its proof). As $b_{\imath} a_{\imath}^{(\alpha)} \leqq u_{\imath}$ for all $\alpha$, in particular, $b_{\imath} a_{\imath}\left(=b_{\imath} a_{\imath}^{\left(\alpha_{0}\right)}\right) \leqq u_{\iota}$, a contradiction. 
LEMMA 10. Let $R$ be an orthogonally complete ring satisfying (A). Let $F=\left\{e_{\Lambda}, \lambda \in \Lambda\right\}$ be an orthogonal set of idempotents in $R$ such that $\phi=x \rightarrow\left(e_{\lambda} x\right)_{\lambda \in \Delta}$ is a monomorphism. Then $\phi$ is an isomorphism.

Proof. For let $x^{(\lambda)} \in e_{\lambda} R, \lambda$ ranging over $\Lambda$. As $X=\left\{x^{(\lambda)}, \lambda \in \Lambda\right\}$ is orthogonal it admits a supremum $x$ in $R$. Evaluating the $\lambda$ th projection of $x$ on $e_{\lambda} R$ we get

$x_{\lambda}=e_{\lambda} x=e_{\lambda}\left(\sup _{\mu \in \Lambda} x^{(\mu)}\right)=\sup _{\mu}\left(e_{\lambda} x^{(\mu)}\right)=\sup \left\{0, e_{\lambda} x^{(\lambda)}\right\}=e_{\lambda} x^{(\lambda)}=x^{(\lambda)}$

for all $\lambda \in \Lambda$. Thus $\phi$ is epi. The lemma is established and implies immediately together with Lemmas 7,8 and 9 the required splitting of $R$.

Remark. As shown by Abian, Property (A) holds for any commutative ring $R$ without nilpotent elements [1, Lemma 8]. Note also that Abian's proof of (A) does not use subdirect representation of $R$.

Acknowledgement. The author thanks the referee for his kind suggestions.

\section{REFERENCES}

1. Alexander Abian, Direct product decomposition of commutative semisimple rings, Proc. Amer. Math. Soc. 24 (1970), 502-507.

2. V. A. Andrunakievič and Ju. M. Rjabuhin, Rings without nilpotent elements, and completely prime ideals, Dokl. Akad. Nauk SSSR 180 (1968), 9-11=Soviet Math. Dokl. 9 (1968), 565-568. MR 37 \#6320.

UNIVERSity OF Windsor, Windsor, Ontario, Canada

Carleton University, Ottawa, Ontario, Canada

Universitł Laval, Québec, Quebec, Canada 